\title{
ASO Visual Abstract: Pathologic N3 Stage (pN3/ypN3) Gastric Cancer-Outcomes, Prognostic Factors, and Pattern of Recurrences after Curative Treatment
}

\begin{abstract}
Anadi Pachaury, MS, MCh ${ }^{1}$, Vikram Chaudhari, MS, DNB ${ }^{1}$, Swati Batra, MS, DNB ${ }^{1}$, Anant Ramaswamy, MD, $\mathrm{DM}^{2}$, Vikas Ostwal, MD, $\mathrm{DM}^{2}$, Reena Engineer, MD, $\mathrm{DNB}^{3}$, Munita Bal, MD, DNB ${ }^{4}$, Shailesh V. Shrikhande, MS, MD, FRCS (Hon) ${ }^{1}$, and Manish S. Bhandare, MS, $\mathrm{MCh}^{1}$

${ }^{1}$ Gastrointestinal and Hepato-Pancreato-Biliary Service, Department of Surgical Oncology, Tata Memorial Centre, Homi Bhabha National Institute, Mumbai, Maharashtra, India; ${ }^{2}$ Department of Medical Oncology, Tata Memorial Centre, Homi Bhabha National Institute, Mumbai, Maharashtra, India; ${ }^{3}$ Department of Radiation Oncology, Tata Memorial Centre, Homi Bhabha National Institute, Mumbai, Maharashtra, India; ${ }^{4}$ Department of Pathology, Tata Memorial Centre, Homi Bhabha National Institute, Mumbai, Maharashtra, India
\end{abstract}

Gastric cancer classified as pathologic stage N3(pN3/ ypN3) behaves aggressively even after the best curative treatment. This retrospective analysis of a prospectively maintained database aimed to assess factors affecting survival and pattern of recurrences and explored possible treatment options to improve outcomes (https://doi.org/10. 1245/s10434-021-10405-3).
Supplementary Information The online version contains supplementary material available at https://doi.org/10.1245/s10434021-10480-6.

DISCLOSURE There are not conflicts of interest.

Publisher's Note Springer Nature remains neutral with regard to jurisdictional claims in published maps and institutional affiliations.

(C) Society of Surgical Oncology 2021

Published Online: 9 August 2021

M. S. Bhandare, MS, MCh

e-mail: manishbhandare@gmail.com 